This item was submitted to Loughborough's Institutional Repository (https://dspace.lboro.ac.uk/) by the author and is made available under the following Creative Commons Licence conditions.

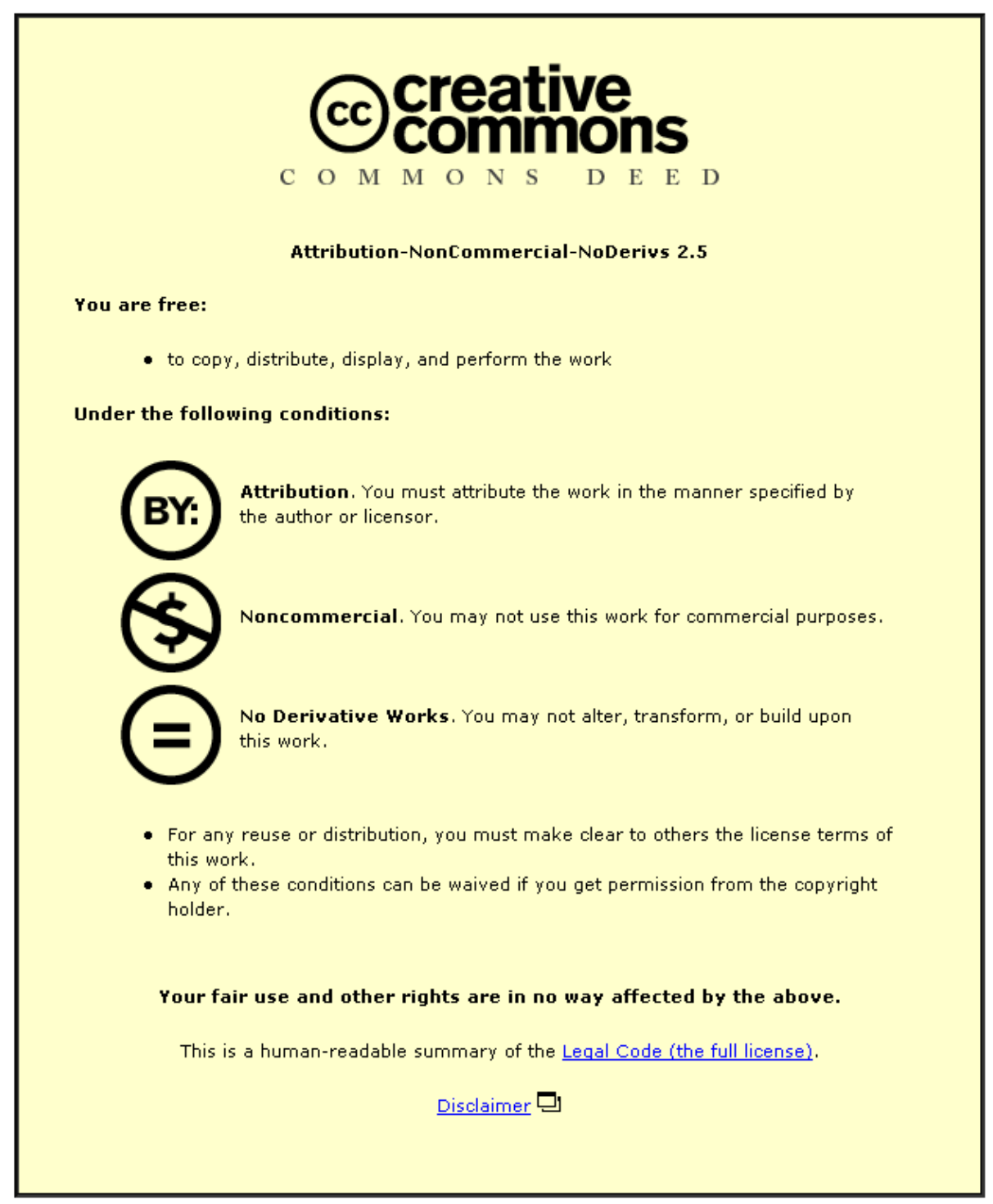

For the full text of this licence, please go to: http://creativecommons.org/licenses/by-nc-nd/2.5/ 


\title{
2000 Conference on Electrical Insulation and Dielectric Phenomena
}

\section{Surface Flashover Dynamics in Metallized Polymer Film Capacitors}

\author{
$M G$ Kong and $Y$ P Lee \\ Department of Electronic and Electrical Engineering, Loughborough University, Leicestershire LE11 3TU, UK
}

\begin{abstract}
Flashover discharges are one of main failure mechanisms in metallized polymer film capacitors. To study the basic characteristics of surface discharges and their dynamics, an equivalent electric circuit model is developed for metallized polymer film capacitors with the surface discharge modelled with a time-dependent resistor. The discharge resistance is related to the thermal dynamic equation, and as such the heat generated within the capacitor unit can be estimated and subsequently used to assess changes in material properties due to temperature rise. Also discussed are the conditions for propagation of surface discharges arc and its elongation. For different combination of key system parameters, surface discharges are stimulated and this provides insights into factors that influence the growth of surface flashover in metallized polymer film capacitors.
\end{abstract}

\section{Introduction:}

There has been significant progress in the technology of metallised polymeric film capacitors over the past 20 years, fuelled by their widespread applications in power systems[1][2]. One of the main drivers in the field is higher energy density with increasingly compact designs. This highlights the need to gain a thorough understanding of breakdown discharges developed within a film capacitors under higher and higher electric stress.

In this paper, we study the evolution of surface flashover discharges with an equivalent circuit model. Temperature gradient between surface plasma and its surrounding space is taken into account by means of heat conduction equations. Numerical examples are then used to provide insights into the fundamental surface flashover processes in film capacitors.

\section{Basic model of surface discharge:}

Appreciable electric field can build up on the surface of capacitor electrodes when the capacitor is switched onto a power system, subjected to an internal breakdown, or simply operated in AC. Such surface electric field can be enhanced at localized surface defects and so may lead to single or multiple surface discharges at the defect area. To ensure the reliability and lifetime of capacitors, it is very important that these surface discharges do not elongate and develop into catastrophic flashover arcs to fail the capacitor.

After a surface discharge is initiated from the surface defect site, it may propagate on the electrode surface. As electric energy required for plasma propagation is likely to be fed via the original defect site, we assume that the surface discharge has a stationary root at the defect site and its other root moves on the electrode surface. Under the influence of the surface electric field, the current leaving the moving root of the plasma may flow along two possible routes on the segmented electrode surface. As illustrated in figure 1, the first route is through successive electrode segments and unbroken fuses linking them whereas the second is through unconnected electrode segments due to either separation gaps or broken fuses between them. If we model electrode segments with surface resistors and separation gaps (or broken fuses) with surface capacitors, the discharge current may be considered to flow through a series of resistors and capacitors along the current path unoccupied by the plasma.

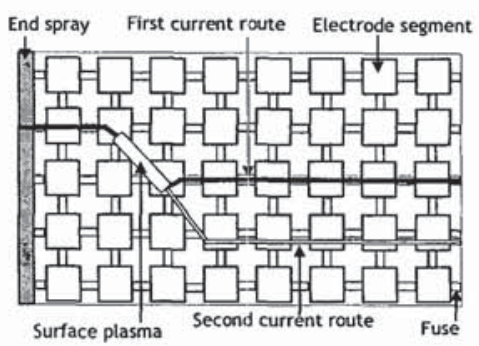

$$
\begin{aligned}
& \text { Surface plasma } \\
&
\end{aligned}
$$

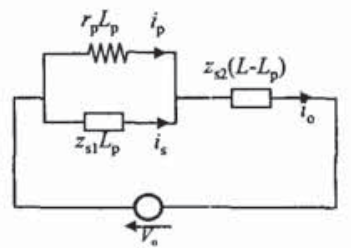

Figure 1 (a) A surface discharge and its current routes on the electrode surface and (b) its equivalent circuit.

As a one-dimensional approximation, the surface discharge is assumed to be of the form of a uniform cylindrical column having a length of $L_{\mathrm{p}}$ and a cross sectional area of $A_{p}$. We further assume that it can be modelled approximately by a resistor with resistivity $\rho_{\mathrm{p}}$ and so gives a discharge resistance of

$$
R_{p}=\rho_{p} L_{p} / A_{p}=r_{p} L_{p}
$$

where $r_{p}$ is the plasma resistance per unit length. The presence of the surface plasma alters the equivalent impedance of the electrode surface along the current path by effectively adding a parallel resistor $R_{p}$ to the surface impedance, $z_{s 1} L_{p}$, of the electrode area of length $L_{p}$ underneath the surface plasma. The current path is then described approximately by the circuit in figure $1 \mathrm{~b}$, where $L$ is the overall length of the current path and $z_{s 2}$ is the per unit length impedance of electrode surface not covered by the surface plasma. 
If $r_{p}>\left|z_{s 1}\right|$, a small fraction of the total surface current, $i_{0}$, will flow through the surface plasma. Hence little power will be supplied to the plasma for its subsequent propagation on the electrode surface, and the plasma is less likely to develop into elongated flashover arcs. If $r_{p}<\left|z_{s 1}\right|$ on the other hand, the plasma will draw sizeable current and can gain sufficient amount of power for its subsequent propagation away from its stationary root. This is the condition under which a surface discharge may elongate and our analysis will be developed.

\section{A circuit model of discharge evolution:}

To bring out the underlying physics of plasma propagation without unnecessary complexity in mathematical formulation, we further assume $r_{p} \ll\left|z_{s 1}\right|$ and so ignore $z_{s 1} L_{p}$ in figure 1b. The plasma resistance is therefore connected in series directly to $z_{s 2}\left(L-L_{p}\right)$, the surface impedance along the current path not covered by the plasma as shown in figure 2. There are two components to the surface impedance, namely a lumped surface resistor, $R_{5}$, representing the total resistance of electrode segments, and a lumped surface capacitor, $C_{s}$, representing the total capacitance of separation gaps, both over the length of $\left(L-L_{\mathrm{p}}\right)$. The circuit in figure 1 reduces to that in figure 2. This is similar to that used for outdoor insulators[3], albeit of the difference in the physical meaning of $R \mathrm{~s}$ and $C \mathrm{~s}$.

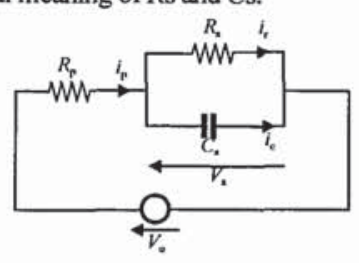

Figure 2 an equivalent circuit model of surface arc.

Let $\rho_{s}$ be the resistivity of electrode material, $\varepsilon_{r}$ the relative permittivity of gap material, and $A_{s}$ the cross sectional area of the gap, we have

$$
\begin{aligned}
& R_{s}=\rho_{s}\left(L-L_{p}\right) / A_{s} \\
& C_{s}=\varepsilon_{0} \varepsilon_{r} A_{s} /\left(L-L_{p}\right)
\end{aligned}
$$

The total impedance of the circuit is therefore

$$
Z_{T}=R_{p}+R_{s} /\left(1+j \omega R_{s} C\right)
$$

with its square modulus as a function of $L_{p}$ given by

$$
\begin{aligned}
\left|Z_{T}\right|^{2}= & v L_{p}^{2}+\rho_{s}^{2} L^{2} / a A_{s}^{2} \\
& \left.+2\left(\rho_{s} / a A_{s}\right) \mid \rho_{p} / A_{p}-\rho_{s} / A_{s}\right] L L_{p} .
\end{aligned}
$$

Here

$$
\begin{aligned}
& a=1+\omega^{2} \varepsilon_{0}^{2} \varepsilon_{r}^{2} \rho_{s}^{2} . \\
& v=\frac{\rho_{p}^{2}}{A_{p}^{2}}+\frac{\rho_{s}^{2}}{a A_{s}^{2}}-\frac{2 \rho_{p} \rho_{s}}{a A_{p} A_{s}}
\end{aligned}
$$

If $r_{p}>\left|z_{s 2}\right|$, the electric field along the surface plasma is greater than that along the current path not covered by the plasma. As the plasma always moves into area of higher electric field[4], the original surface discharge may not propagate when $r_{p}>\left|z_{s 2}\right|$ and so the condition for plasma propagation is $r_{p}<\left|z_{s 2}\right|$. Specifically for the above circuit equations, it is

$$
d\left|Z_{T}\right| / d L_{p}<0
$$

From eq.(4), the derivative of $\left|Z_{T}\right|^{2}$ with respect to $L_{p}$ is

$$
\frac{d\left|Z_{T}\right|^{2}}{d L_{p}}=2 v L_{p}-2 \frac{\rho_{s}}{a A_{s}}\left[\frac{\rho_{s}}{A_{s}}-\frac{\rho_{p}}{A_{p}}\right] L
$$

so the plasma propagation condition of eq.(6) becomes

$$
\frac{L_{p}}{L}<\frac{\rho_{s}}{a v A_{s}}\left[\frac{\rho_{s}}{A_{s}}-\frac{\rho_{p}}{A_{p}}\right] .
$$

Note that $r_{p}<\left|z_{32}\right|$ implies

$$
r_{p}<\rho_{s} /\left(A_{s} \sqrt{a}\right)=r_{s} / \sqrt{a}
$$

according to eq.(3). Since $a>1$, eq.(9) suggests that

$$
r_{s}>\sqrt{a} r_{p}>r_{p}
$$

and so the right hand side of eq.(8) is always positive.

The maximum plasma length is $L$ when the surface discharge develops into a flashover arc across the entire current route. So $0 \leq L_{p} / L \leq 1$. If in (8)

$$
\frac{\rho_{s}}{a v A_{s}}\left[\frac{\rho_{s}}{A_{s}}-\frac{\rho_{p}}{A_{p}}\right] \geq 1
$$

then the plasma propagation condition of (8) is always satisfied. Thus it is possible for the plasma to develop into a full-scale flashover. With eq.(5), (11) becomes

$$
r_{p} \leq r_{s} / a \text {. }
$$

Since $a>1$, eq.(12) represents a stronger and more specific condition for arc propagation than the condition of eq.(9). If, on the other hand,

$$
\frac{\rho_{s}}{a \cup A_{s}}\left[\frac{\rho_{s}}{A_{s}}-\frac{\rho_{p}}{A_{p}}\right]<1
$$

$L_{p}$ will always be less than $L$. This suggests that the discharge may elongate to a point within the electrode boundary at which it will extinguish. With the aid of (9), condition (13) leads to

$$
r_{s} / a<r_{p}<r_{s} / \sqrt{a} .
$$

Inequality (14) specifies the condition under which the surface plasma may propagate but extinguish before it reaches the electrode boundary.

\section{Heat dissipation of the surface plasma:}

Inequalities (12) and (14) express conditions for plasma elongation and its development into flashover arc in terms of surface impedance and plasma resistance. As the plasma propagates on the electrode surface, its temperature changes and so (12) and (14) need to be assessed at each possible temperature. More 
importantly the above analysis does not take into account the temperature gradient between the plasma column and its surrounding space, which may lead to plasma propagation due to heat conduction.

The surface plasma is sustained by the surface current, which is in turn supplied by either capacitor energy or external power supply. Without heat exchange with its surrounding area, the plasma will be heated up through its own ohmic losses, $i_{p}^{2} R_{p}$, and as such its temperature will rise. However as it interacts thermally with the surrounding area, its temperature reduces in the form of heat dissipation and radiation into the surrounding capacitor space. Therefore there are two competing events for plasma temperature change. If its net temperature change is positive, the plasma becomes less resistive and according to conditions in (12) and (14) its development into an elongated flashover will become more probable. If the net temperature change is negative, the plasma will become increasingly resistive and less likely to elongate. Thus when there is no net temperature change, the plasma resistance remains unchanged and this represent the critical condition under which plasma elongation is not encouraged thermally.

In general heat conduction is governed by [5]

$$
\rho_{m} c_{p} \frac{\partial T}{\partial t}=k \frac{\partial^{2} T}{\partial x^{2}}+q^{*}
$$

in the one-dimensional limit. Here $\rho_{m}$ is the density, $c_{p}$ the specific heat, $k$ thermal conductivity, $x$ the distance along the current route from a predefined origin point, and $q^{*}$ the internally generated heat per unit volume. As an approximation, radiation loss of the plasma is assumed to be negligible and so $q^{*}=i_{p}{ }^{2} R_{p} / A_{p} L_{p}$ is the internal heat source for the plasma column. Furthermore we assume that the $\partial T / \partial t$ term on the left hand side of the equation is predominately controlled by $q^{*}$ and so the spatial variation of temperature along the plasma is neglected. Because surface plasmas are often sparks of short length, this is a reasonable approximation. Thus for the plasma column

$$
\frac{\partial T_{p}}{\partial t}=\frac{i_{p}^{2} r_{p}}{\rho_{m p} c_{p} A_{p}}=g_{1}\left(T_{p}\right) .
$$

The current following through the plasma is related to the external voltage $V_{0}$ via the circuit impedance of eq.(3). Note that $r_{s}$ is usually between $50-1000 \Omega / \mathrm{cm}$ and $C_{s}=1-5 \times 10^{-18} \mathrm{~F}[6]$. Therefore for $\omega R_{s} C_{s}$ to be comparable to unity, the frequency needs to be well above $1 \mathrm{THz}$. In other words, $a \approx 1$ for frequencies well below $1 \mathrm{THz}$ and so $Z_{T}=R_{p}+R_{s}$. Consequently

$$
g_{1}(T)=\frac{E_{0}^{2} r_{p}(T) / \rho_{m p}(T) c_{p}(T) A_{p}}{\left[r_{s}+\left(r_{p}(T)-r_{s}\right)\left(L_{p} / L\right)\right]^{2}}
$$

where $E_{0}=V_{0} / L$ is the average surface electric field.

For the current path on the non-plasma electrode surface, its temperature rise is largely induced by heat conduction from the plasma column and the ohmic heating should be negligible. In other words, the nonplasma section of the current path acts as a heat sink for the plasma column. Thus eq.(15) becomes

$$
\rho_{m s} c_{p s} \frac{\partial T_{s}}{\partial t}=k_{s} \frac{\partial^{2} T_{s}}{\partial x^{2}} .
$$

Suppose the non-plasma section of length $x_{0}$ has one end, at $x=x_{0}$, attached to the plasma column of temperature $T_{p}$ and its other end, at $x=0$, maintained at ambient temperature of $T_{a m b}$. Eq.(18) can be solved analytically [5], and one solution is

$$
T_{s}=T_{a m b}+\left(T_{p}-T_{a m b}\right) \sin \left[\frac{\pi x}{2 x_{0}}\right] e^{-\left(\pi / 2 x_{0}\right)^{2} \alpha_{s} t}
$$

where $\left.\alpha_{x}=k_{s}\right\lrcorner \rho_{m s} c_{p x}$ is the thermal diffusivity of the electrode material. At the plasma interface with nonplasma section, the temperature is reduced according to

$$
T_{s}=T_{a m b}+\left(T_{p 0}-T_{o m b}\right) e^{-\left(\pi / 2 x_{0}\right)^{2} \alpha, t}
$$

where $T_{p 0}$ is the plasma temperature $t=0$.

At room temperature, $\alpha_{s}=0.06-0.08 \mathrm{~m}^{2} / \mathrm{s}$ for pure aluminium and some of its alloy forms[5]. If we use the half width of the capacitor electrode for $x_{0}$ at, say, $8 \mathrm{~cm}$ (implying an elongated plasma of $8 \mathrm{~cm}$ long), then $\left(\pi / 2 x_{0}\right)^{2} \alpha_{s} t \approx 30 t$. As a typical discharge event lasts well within the milli-second range, the above term very small and so

$$
T_{s}-T_{p 0} \approx-\left(T_{p 0}-T_{a m b}\right)\left(\pi / 2 x_{0}\right)^{2} \alpha_{s} t
$$

If the temperature reduction of eq.(21) due to heat conduction at the moving root of the plasma is the same as its temperature rise of eq.(16) due to ohmic heating, the plasma will be in thermal equilibrium and its temperature remain unchanged. Otherwise there is a net temperature change along the plasma column. To this end, we introduce the ratio of temperature change

$$
f(T) \equiv \frac{\left|\Delta T_{\text {rise }}\right|}{\left|\Delta T_{\text {drop }}\right|}=\frac{g_{1}(T)}{\left(T-T_{\text {amb }}\right)\left(\pi / 2 x_{0}\right)^{2} \alpha_{s}}
$$

Note that $x_{0}=L-L_{p}$ and so eq.(22) reduces to

$$
f(T)=\frac{\left(1-L_{p} / L\right)^{2}}{\left[1+\left(r_{p} / r_{s}-1\right) L_{p} / L\right]^{2}} \Theta(T)
$$

where

$$
\Theta(T)=\frac{L^{2}}{A_{p}}\left[\frac{2}{\pi}\right]^{2} \frac{E_{0}^{2} r_{p} / r_{s}^{2}}{c_{p} \rho_{m p} \alpha_{s}\left(T-T_{a m b}\right)} .
$$

The above equation is a measure of the relative temperature increase of the plasma column in the onedimensional limit, in which the plasma column is assumed to have the same cross sectional area as the film capacitor. As the initial surface spark discharge is usually short and filamentary, this is an overestimate of the thermal impact of the plasma. To account for this, the plasma temperature rise of eq.(16) is reduced by the ratio of the cross sectional 
area of the plasma column, $A_{p}$, and that of a film capacitor, $d_{c} \times L_{w}$, with $d_{c}$ being the thickness of the capacitor and $L_{w}$ the width of the film. Furthermore as heat conduction of the plasma is also possible along its length, an additional coefficient of $d_{p} L_{p} / d_{c} L$ should be introduced with $d_{p}$ being the plasma diameter. If we assume $L_{w}=L$ for a square film, eq.(24) becomes

$$
\Theta(T)=\frac{4}{\pi^{2}} \frac{d_{p} L_{p}}{d_{c}^{2}} \frac{E_{0}^{2} r_{p} / r_{s}^{2}}{c_{p} \rho_{m p} \alpha_{s}\left(T-T_{a m b}\right)} .
$$

If $\Theta(T)>1$, the temperature rise of the plasma column cannot be compensated by the temperature reduction due to its heat conduction to the non-plasma area. Hence the plasma becomes hotter and it becomes less resistive leading to its elongation into its surrounding space. If $\Theta(T)<1$, the temperature rise of the plasma column is well offset by the temperature reduction of the nonplasma area and as such the plasma becomes progressively cooler eventually extinguished.

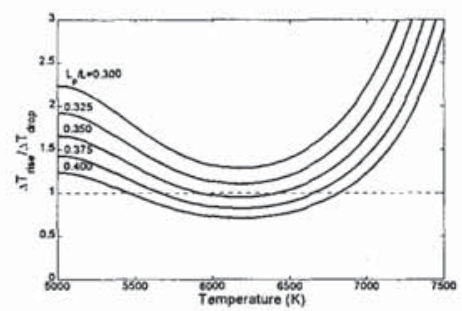

Figure 3 Temperature rise against initial plasma temperature

To illustrate the implication of eq.(23), we consider a film capacitor with $E_{0}=200 \mathrm{~V} / \mathrm{cm}, T_{\text {amb }}=298 \mathrm{~K}, r_{s}=$ $200 \Omega / \mathrm{cm}$, and $d_{p}=2 \mu \mathrm{m}$. For practical multiple layer film capacitors, $d_{c}=2-5 \mathrm{~cm}$, and for all cases discussed here we choose $d_{c}=2.5 \mathrm{~cm}$. Specific heat, temperature, and electrical resistivity of air plasmas are obtained from [7]-[9] and diffusivity of aluminium is obtained from [10]. In figure 3 , the normalised temperature rise of the plasma column is plotted as a function of its initial temperature. If the plasma length is initially less than $32.5 \%$ of the capacitor length, its temperature rise is always greater than the temperature reduction due to heat conduction. As a result, it becomes hotter and less resistive. This leads to the plasma column drawing greater current from the surface electric field and it will grow in length and elongate. As the plasma column elongates, its temperature rise becomes smaller and eventually less than the conduction induced temperature reduction. At this point, a thermal equilibrium is reached and the plasma will maintain its length. However in the event of a reduction in surface electric field, the plasma will subsequently undergo extinction. In the case of figure 3 , the initial plasma will elongate but extinguish at a length just over $35 \%$ of the capacitor length. This is of course a significant surface flashover. In practice, surface electric field is often time-dependent either especially when induced by an internal breakdown or a time-dependent external source. Plasma elongation should complete much earlier, and figure 3 represents the worse scenario.

Also of interest is that the plasma resistance used in figure 3 is much higher than surface resistance. Therefore the significant temperature gradient between the plasma column and its surrounding space results in an overwhelming heat conduction effect on plasma propagation and the electric field based argument of (6) becomes irrelevant. In other words, condition (12) and (14) are valid only when the temperature gradient is negligibly small.

\section{Conclusion:}

We have developed an equivalent circuit model to study the propagation and elongation of surface discharges in polymeric film capacitors. Thermal effects are also included in a one-dimensional heat transfer model. It has been shown that if the temperature of the initial surface plasma is sufficiently close to the ambient temperature a simple plasma elongation criterion can be established based on plasma resistance and surface resistance. However when there is considerable temperature gradient between the plasma and its surrounding space, the simple resistance based criterion becomes invalid as the problem becomes dominated be thermal events.

\section{Acknowledgement:}

YPL acknowledges financial support from ABB Power T \& D, Capacitor Division, UK, and Loughborough University.

\section{Reference:}

[1] D. G. Shaw et al, "A changing capacitor technology- failure mechanism and design innovations", IEEE Trans Electrical Insulation, vol.16, pp. $399-413,1981$

[2] C. W. Reed and S. W. Chichanowski, "The fundamentals of ageing in HV power film capacitors", IEEE Trans Dielectric and Electrical Insulation, vol.1, pp. $904-922,1994$.

[3] N. Dhahbi-Megriche, A. Beroual, and L. Krahenbuhl, "A new proposal model for flashover of polluted insulators"J. Phys. D: Applied Physics, Vol.30, pp. 889-894, 1997.

[4] H. Boehme and F. Obenaus, "Pollution flashover tests on insulators in the laboratory and in systems and the model concept of creepage-path flashover", CIGRE Paper 407, 1966.

[5] A. J. Chapman, Heat Transfer, 3rd Ed, MacMillan, New York, 1974.

[6] G. J. Walters, "High energy metallized films and capacitor thereof", private communications.

[7] S. V. Dresvin, Physics and technology of low-temperature plasmas, The Iowa State University Press, Ames, 1977.

[8] R. N. Gupita et al, Calculations and cune fits of thermodynamic and transport properties for equilibrium air to $30000 \mathrm{~K}$. NASA Reference Publication 1260, 1991

[9] H. Kroepelin et al, Thermodynamic diagrams for high temperature plasmas of air, aikcarbon, carboninydrogen mixtures, and argon, Pergamon Press, Oxford, 1971.

[10] Y. S. Touloukian et al, Thermophysical Properties of Matter. Volume 10: Thermal Diffusivity, 1973. 